\title{
Acute-on-chronic liver failure: A predictor of poor prognosis in patients with variceal bleeding or a risk factor for variceal bleeding?
}

\author{
Nae-Yun Heo \\ Department of Internal Medicine, Inje University College of Medicine, Busan, Korea
}

Keywords: Acute-on-chronic liver failure; Esophageal varix; Bleeding

\section{See Article on Page 540}

Acute-on-chronic liver failure (ACLF) is a dangerous condition defined by organ failure and increased short-term mortality. Therefore, it is not surprising that ACLF is associated with higher mortality after variceal bleeding in cirrhotic patients. However, limited direct data are available regarding this issue.

In this issue of Clinical and Molecular Hepatology, Shin et al.' investigated whether the diagnosis of ACLF after variceal bleeding was associated with subsequent survival. The prevalence of ACLF was $13 \%$ among patients with acute deterioration of chronic liver disease following acute variceal bleeding. The overall survival of patients with ACLF was lower compared to those without ACLF, and a higher ACLF grade was related to lower overall survival. Recently, Trebicka et al. ${ }^{2}$ first reported that $17.8 \%$ of 2,138 patients with acute variceal bleeding had ACLF at admission, and that the presence of ACLF was independently related to rebleeding and mortality. Therefore, we assumed that $10-20 \%$ of cases involving acute variceal bleeding may experience ACLF at admission or dur-

\section{Abbreviations:}

ACLF, acute-on-chronic liver failure; TIPS, transjugular intrahepatic portosystemic shunt ing hospitalization, suggesting the need for careful evaluation of patients with variceal bleeding. The diagnosis of ACLF requires adequate management by physicians to reverse the clinical course.

According to Shin et al.," the 28-day cumulative mortality was as high as $41 \%$ in patients with variceal bleeding and ACLF, which indicates a very high risk of mortality in this group. However, the mortality of patients with variceal bleeding in case of decompensated cirrhosis might be higher than that in the former. In the study population of Shin et al., ${ }^{1}$ these patients were excluded. Therefore, we could not compare the mortality between the two groups. Based on a clinical perspective, it is sometimes difficult to differentiate ACLF from aggravation of decompensated cirrhosis. Although ACLF is defined by acute aggravation in patients with reversible liver function, the prognosis of ACLF grade 3 is poor and may be irreversible. In this study, patients with variceal bleeding and ACLF grade 3 showed very poor overall survival $<10 \%$ in 90 days). Therefore, liver transplantation is imperative in such cases similar to aggravated decompensated cirrhosis.

Unfortunately, this study did not suggest the optimal treatment option for variceal bleeding in ACLF patients in their cohort due to
Corresponding author : Nae-Yun Heo

Department of Internal Medicine, Inje University College of Medicine, 875 Haeun-daero, Haeundae-gu, Busan 48108, Korea

Tel: +82-51-797-0200, Fax: +82-51-797-1340

E-mail:nyheo@hanmail.net

https://orcid.org/0000-0001-6571-8935 
limited database. The clinical course may be changed if early transjugular intrahepatic portosystemic shunt (TIPS) is used to control active variceal bleeding or secondary prevention of rebleeding. Several studies showed that patients with early or pre-emptive TIPS had a lower rebleeding risk and higher overall survival, even in ACLF patients. ${ }^{2-4}$ However, the aggravation of liver failure in severe decompensated cirrhosis still remains a concern. Lv et al. ${ }^{4}$ reported that early TIPS with covered stents improved transplantation-free survival in selected patients with advanced cirrhosis (Child B or C) and acute variceal bleeding compared to standard treatments, such as endoscopic band ligation and beta-blockers following salvage TIPS as needed. The proportion of ACLF cases in their study is unknown. Therefore, further studies are needed to determine the indications for early TIPS in ACLF patients.

Finally, it is unclear whether ACLF induces variceal bleeding or vice versa in each case. In patients with ACLF, hepatic venous pressure gradient was elevated, which increases the risk of variceal bleeding. Also, in case of variceal bleeding, subsequent hypoxic hepatitis may induce liver and renal failure and hepatic encephalopathy in chronic liver disease. ${ }^{5}$ According to Joshi et al. ${ }^{6}{ }^{6}$ variceal bleeding was the most frequent morbidity accompanying ACLF among $30 \%$ of the patients in their cohort. Both studies by Shin et al. ${ }^{1}$ and Trebicka et al. ${ }^{2}$ did not reveal the temporal relationship between variceal bleeding and ACLF. Therefore, we could not compare the survival according to the order of the two pathologic conditions. Generally, the baseline characteristics of patients with underlying ACLF followed by variceal bleeding might be poorer than those of patients manifesting ACLF after variceal bleeding. However, the precise prognosis of both groups remains unknown, and requires further analysis.

The greatest limitation of Shin's study was the lack of information related to technical issues involving endoscopic treatment. Due to coagulopathy and cytopenia, variceal bleeding is sometimes difficult to control via endoscopic approach. Hemodynamic instability in ACLF may interfere with endoscopy due to hypoten- sion. Early access and advances in endoscopic approach might reduce the risk of mortality in ACLF associated with variceal bleeding.

ACLF is probably a predictive factor of poor prognosis after variceal bleeding, based on the results of recent and current studies. Regardless of the order of events, it is possible that ACLF and variceal bleeding may influence each other with negative synergism, which facilitates the clinical monitoring of patients for possible multi-organ failure or bleeding events.

\section{Conflicts of Interest}

The authors have no conflicts of interests to disclose.

\section{REFERENCES}

1. Shin J, Yu JH, Jin YJ, Yim HJ, Jung YK, Yang JM, et al. Acute-onchronic liver failure as a major predictive factor for mortality in patients with variceal bleeding. Clin Mol Hepatol 2020;26:540-553.

2. Trebicka J, Gu W, Ibáñez-Samaniego L, Hernández-Gea V, Pitarch C, Garcia E, et al. Rebleeding and mortality risk are increased by ACLF but reduced by pre-emptive TIPS. J Hepatol 2020:S01688278(20)30236-1.

3. García-Pagán JC, Caca K, Bureau C, Laleman W, Appenrodt B, Luca $A$, et al. Early use of TIPS in patients with cirrhosis and variceal bleeding. N Engl J Med 2010;362:2370-2379.

4. Lv Y, Yang Z, Liu L, Li K, He C, Wang Z, et al. Early TIPS with covered stents versus standard treatment for acute variceal bleeding in patients with advanced cirrhosis: a randomised controlled trial. Lancet Gastroenterol Hepatol 2019;4:587-598.

5. Al-Mahtab M, Akbar SM, Garg H. Influence of variceal bleeding on natural history of ACLF and management options. Hepatol Int 2016;10:436-439.

6. Joshi D, O'Grady J, Patel A, Shawcross D, Connor S, Deasy N, et al. Cerebral oedema is rare in acute-on-chronic liver failure patients presenting with high-grade hepatic encephalopathy. Liver Int 2014;34:362-366. 